\title{
Geospatial modeling to identify the effects of anthropogenic processes on landscape pattern change and biodiversity
}

\begin{abstract}
This research used geospatial data to quantify biodiversity changes and landscape pattern change to track anthropogenic impacts of such changes at the Mouteh Wildlife Refuge (MWR), Isfahan, Iran. Satellite image duration of four decades, LandSat1-5, and IRS-P6 data were used to develop land cover classification maps for 1971, 1987, 1998, and 2011. The number and size of land cover patches, the degree of naturalness, and the diversity indices were calculated and compared for a 40 -year period. The results showed an increasing concern with regard to unplanned human activities. Some improvements of the natural landscape also occurred in the core protected zone of the study area. The number and size of land cover patches, the degree of naturalness, and the diversity indices were calculated. Overall changes in natural land use between 1971 and 1998 at MWR showed that the number of patches for natural land use has increased, but it also showed a decrease in 2011. Similar changes were observed for seminatural land use. Within the artificial classes, the number and area of patches were higher and the largest patch occurred in 2011. The maximum variation of diversity is related to the year 2011. The results showed an increasing concern with regard to unplanned human activities. Some improvements of the natural landscape also occurred in the core protected zone of the study area. Remote sensing and geographic information system offers an important means of detecting and analyzing temporal changes occurring in our landscape.
\end{abstract}

Keyword: Landscape ecology; Naturalness; Diversity; GIS; Mouteh Wildlife Refuge 